\title{
Dental and Anaesthetic Challenges in a Patient with Dystrophic Epidermolysis Bullosa
}

$$
\text { تحديات طب الأسنان والتخدير لمريض مصاب بانحلال البشرة الفقاعي الحثلي }
$$

$$
\text { علي العبادي، صلاح الدين العزري، عبدالعزيز بـاكثير، يسرى الريامية }
$$

\begin{abstract}
Epidermolysis bullosa is a group of rare genetic disorders characterised by skin and mucous membrane fragility and systemic manifestations of variable severity. We report a case of dystrophic epidermolysis bullosa in an 18-year-old male patient who presented to the Department of Oral Health at Sultan Qaboos University Hospital, Muscat, Oman, in 2015 with recurrent dental pain and infections. Due to the poor dental status of the patient and anticipated operative difficulties due to microstomia and limited mouth opening, the patient underwent full dental clearance under general anaesthesia. This article discusses the dental and anaesthetic challenges encountered during the management of this patient and provides a brief literature review.
\end{abstract}

Keywords: Dystrophic Epidermolysis Bullosa; Dentistry; Disease Management; General Anesthesia; Case Report; Oman.

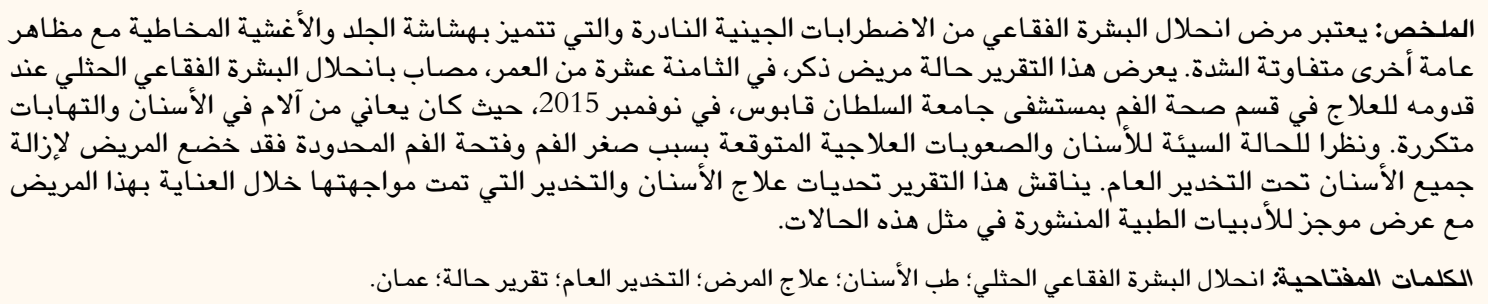

$\mathrm{E}$

PIDERMOLYSIS BULLOSA (EB) IS A GROUP OF genetic vesiculobullous disorders which are characterised by skin and mucous membrane fragility and systemic manifestations of variable severity ${ }^{1,2}$ Affected individuals usually present with continuous blister formation due to mechanical trauma or heat; in some cases, the blisters even develop spontaneously without any apparent cause. ${ }^{3,4}$ The onset of the disease usually occurs immediately after birth up until five years of age. ${ }^{5}$ Four major forms of EB have been described, depending on the level of skin involvement: EB simplex (EBS), which involves the intra-epidermal layer; junctional EB (JEB), which involves the lamina lucida; dystrophic EB (DEB), which involves the sublamina densa; and Kindler syndrome, which involves multiple layers of the epidermis. These types are further subdivided depending on the severity of the condition, mode of inheritance and the genes involved. ${ }^{2}$ The risk of morbidity and mortality among EB patients is high. ${ }^{6}$

The prevalence of EB worldwide is variable.? In Saudi Arabia, Abahussein et al. reported the prevalence of EBS, JEB and DEB to be $1.7 \%, 0 \%$ and
$3.7 \%$ per million, respectively; moreover, parental consanguinity was noted among $87.5 \%$ of cases. ${ }^{8}$ There are no published studies on the exact prevalence of EB in Oman; however, unpublished data from national dermatology units indicate approximately 60 registered cases. This report focuses on the dental and anaesthetic challenges encountered during the management of an Omani adult patient with severe DEB.

\section{Case Report}

An 18-year-old male was referred to the Department of Oral Health at Sultan Qaboos University Hospital, Muscat, Oman, in 2015 for the management of recurrent dental pain and localised dental infections. The patient had been seen by many general dental practitioners in different institutions over the previous two years. He had been managed conservatively with multiple courses of antibiotics, which were successful in temporarily controlling his symptoms. In early childhood, the patient had been diagnosed with DEB and had undergone multiple surgeries under general 

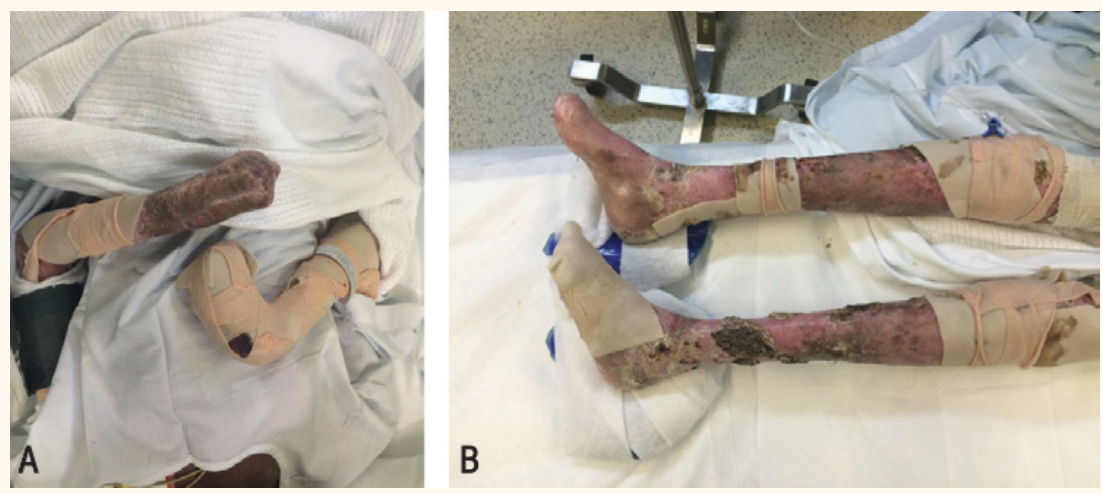

Figure 1: Limb deformity, scarring with contractures and pseudosyndactyly of the (A) upper and (B) lower limbs of an 18-year-old male patient with dystrophic epidermolysis bullosa. Note the intravenous cannula on the right thigh secured with non-adhesive paper tape and bandages to prevent tearing and blistering.

anaesthesia (GA) at different hospitals, including balloon dilatation of oesophageal strictures, incisional release of pseudosyndactyly and insertion and removal of a percutaneous endoscopic gastrostomy tube. He applied silver sulfadiazine ointment on the skin lesions and used artificial tears on a daily basis. He had no known allergies and his diet was limited to soft and puréed food. The parents reported that they were first cousins and that he had three other siblings, the youngest of whom was a three-year-old male also affected by DEB while the other two siblings were healthy.

At referral, the patient was accompanied by his parents and appeared to have normal cognitive function. He was initially anxious and uncooperative during the examination due to severe dental pain. His height was $1.44 \mathrm{~m}$ and his weight was below the $3^{\text {rd }}$ percentile for his age $(27.7 \mathrm{~kg}$; body mass index: $13.4 \mathrm{~kg} / \mathrm{m}^{2}$ ). His vital signs were within normal limits. Most of his skin was affected by generalised body blisters, although some were in the healing stage. He also had pseudosyndactyly of the hands and feet, loss of the toenails and fingernails and extensive skin contractures and scarring. Most of his upper and lower limbs were covered with protective medicated gauze and bandages [Figure 1]. An oral examination

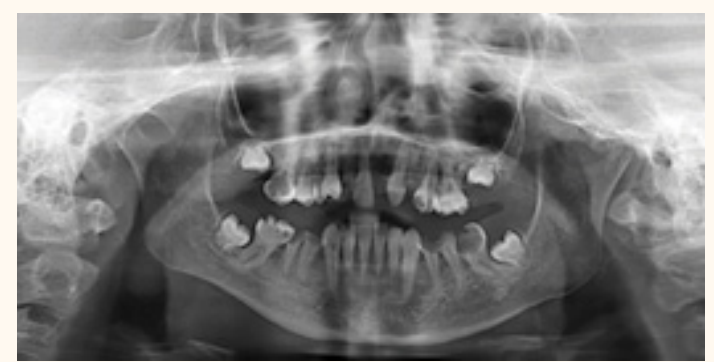

Figure 2: An orthopantomogram of an 18-year-old male patient with dystrophic epidermolysis bullosa showing multiple grossly carious teeth with severe bone loss in the lower anterior teeth. was conducted with difficulty due to microstomia and limited mouth opening. The patient had ankyloglossia and a smooth tongue with obliteration of the buccal and lingual vestibules. His dental status was poor, with multiple advanced dental caries in most of his teeth; these had caused the formation of numerous dental sinuses which discharged to the oral cavity. Periodontally, there was involvement of the lower anterior teeth with increased mobility and alveolar bone loss [Figure 2]. Due to the patient's poor dental status and prognosis, in addition to anticipated operative difficulties due to the microstomia and limited mouth opening, a full dental clearance under GA was recommended. The appropriate written consent for the procedure was obtained after comprehensive consultation with the patient and his parents.

A pre-anaesthetic evaluation revealed that airway control and the maintenance of skin integrity were two major concerns. Intravenous (IV) access and endotracheal intubation procedures were anticipated to cause difficulties and potentially increase the risk of trauma to the patient. As such, preoperative blood investigations were avoided. In the operating room, a full-bed-size soft sheet was placed on the operating table to avoid skin friction and the room temperature was increased to avoid hypothermia. The patient was allowed to move himself slowly onto the operating table and all pressure points were supported with padding and bandages. A clip-on pulse oximetry probe was attached to the right ear lobe to monitor capillary oxygen saturation [Figure 3A]. Defibrillator pads were used to attach the electrocardiography (ECG) electrodes to the chest and a non-invasive blood pressure cuff was applied after wrapping the arm with cotton pads [Figure 3B]. All airway equipment was generously lubricated before touching the mucosa.

The anaesthesia was induced using sevoflurane and a $50 \%$ oxygen to $50 \%$ nitrous oxide mixture. The IV 


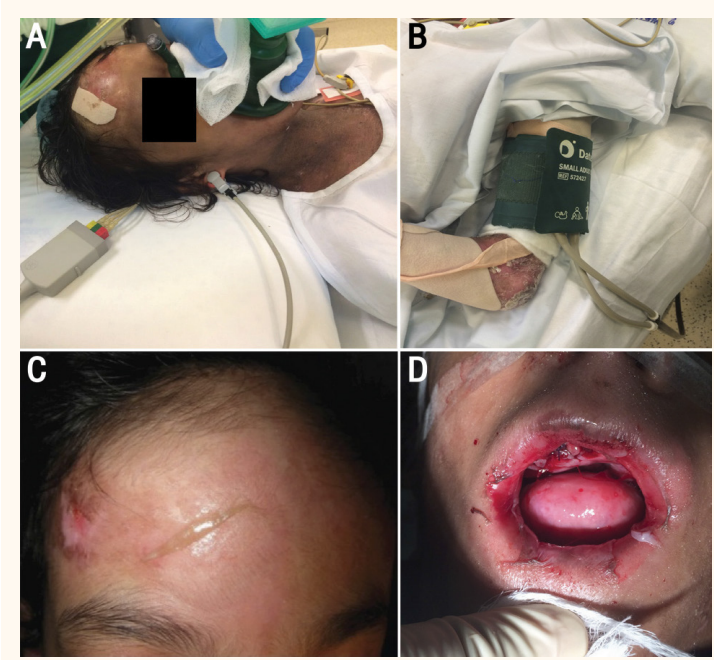

Figure 3: Intraoperative photographs of an 18-yearold male patient with dystrophic epidermolysis bullosa showing (A) a clip-on oxygen saturation probe attached to the ear lobe and (B) a blood pressure cuff applied over a protective cotton pad to avoid skin damage during a full dental clearance under general anaesthesia. However, after completion of the surgery, there was evidence of $(\mathbf{C})$ forehead skin sloughing due to the use of a face mask during intubation and (D) mucosa and skin sloughing of the lips and perioral tissues.

access was secured in the right great saphenous vein and blood was collected and sent for investigations. Subsequently, the anaesthesia was supplemented with fentanyl, propofol and rocuronium. A C-MAC ${ }^{\circledR}$ videoassisted laryngoscope (C-MAC Electromag, Ronse, Belgium) was used to reduce the number of attempts at intubation and the vocal cords were visualised without too much opening or manipulation of the mouth. An atraumatic nasal endotracheal intubation was performed with a small $5.5 \mathrm{~mm}$ non-cuffed endotracheal tube after being softened with a warm saline solution and adequate lubrication. Non-adhesive paper tape was used to secure the endotracheal tube and IV catheter. The eyes were lubricated with ophthalmic ointment and covered lightly with soft gauze and paper tape. The anaesthesia was maintained with sevoflurane and the analgaesia was supplemented intravenously with paracetamol. The patient was kept warm with a Bair Hugger ${ }^{\mathrm{TM}}$ intraoperative warming blanket (3M Company, Maplewood, Minnesota, USA) and his temperature was monitored using an axillary probe. Reasonable oral access was achieved via nasal intubation but was limited by the microstomia. Intraoperatively, local anaesthesia was avoided to prevent mucosal injury and the dental extractions were done as atraumatically as possible in order to minimise injury to the oral tissues. The sockets were packed with haemostatic gauze (Surgicel ${ }^{\circledR}$, Ethicon Inc., Somerville, New Jersey, USA) and suturing was kept to a minimum to avoid soft tissue trauma and postoperative bleeding. At the end of the surgery, the patient was extubated uneventfully with no major anaesthetic complications. Despite the frequent intraoperative use of hydrocortisone lubrication on the lips and perioral tissues, sloughing of the skin of the forehead [Figure 3C] as well as the oral and lip mucosa [Figure 3D] was observed.

Following the procedure, the patient recovered well and was prescribed augmentin and paracetamol. During the postoperative period, regular lip lubrication, antiseptic chlorhexidine mouth-rinsing and an adequate soft diet was maintained. The blood investigations revealed iron deficiency anaemia and the patient was subsequently prescribed iron supplements. All of the other blood investigation results were within normal limits. The patient was discharged on the third postoperative day. At a four week follow-up, the soft tissues and extraction sockets had healed satisfactorily with minimal scarring [Figure 4]. At the time of writing, the patient was currently in the process of undergoing prosthetic dental rehabilitation.

\section{Discussion}

Genetic mutations can result in the production of defective or absent proteins important to the integrity of the skin and mucosa. ${ }^{9}$ The phenotypic presentation of DEB varies from mild, seen mostly in the dominant type of DEB, to the generalised severe scarring form, seen mostly in the recessive type. ${ }^{10}$ The patient in the present case showed clinical features of DEB arising due to a mutation in the collagen type VII alpha 1 gene, which leads to reduced epidermal adherence. ${ }^{9}$ As both parents were unaffected first cousins and the patient had one affected and two healthy siblings, it is likely that he had autosomal recessive DEB.

Oral manifestations of DEB include recurrent oral blisters and erosions, which heal with scarring and eventually lead to microstomia, ankyloglossia, smooth tongue and vestibular obliteration, as observed in the present case. ${ }^{3,11}$ Many published reports have highlighted that DEB patients are at high risk of developing rampant caries due to enamel defects, inadequate oral hygiene caused by recurrent painful oral blisters and loss of manual dexterity and a highcalorie cariogenic diet designed to compensate for their high metabolic state.,12,13 In the current case, these features led to the extensive destruction of the dentition and recurrent episodes of severe dental pain and infections. Oral and dental features of DEB present a challenge when providing dental treatment. Treatment conducted under local anaesthesia is not widely practised due to microstomia and limited 


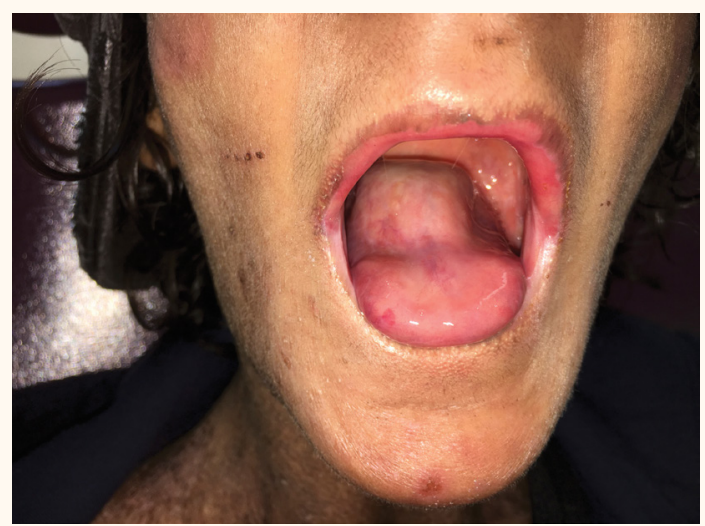

Figure 4: Postoperative photograph of an 18-year-old male patient with dystrophic epidermolysis bullosa four weeks after a full dental clearance showing satisfactory healing of the oral tissues and dental sockets, with minimal scarring.

mouth opening, in addition to the risk of blister formation and soft tissue injury following administration of the anaesthetic. ${ }^{1,14}$ Conducting treatments under GA is justifiable and widely reported in the literature, especially if extensive dental treatment is required. ${ }^{1,13-16}$ In the present case, the dental clearance was performed under GA due to the relatively extensive nature of the disease as well as to minimise potential trauma to the oral tissues and reduce associated stress and anxiety on the part of the patient.

Systemic manifestations of DEB can also complicate the administration of GA and pose anaesthetic challenges in terms of IV access, monitoring of vital signs and intubation. ${ }^{15,16}$ Extensive skin blistering and scarring with limb contractures can conceal venous access and the central venous route may be necessary as a last resort. ${ }^{17}$ Placing adhesive dressings for IV lines and ECG pads directly onto the skin can lead to tearing and blistering. ${ }^{13,14}$ In the present case, non-adhesive defibrillator pads were attached to adhesive ECG pads. Extensive skin deformities and pseudosyndactyly can also present a challenge when monitoring oxygen saturation; as in the current case, a clip-on probe can be attached to the ear lobe to circumvent this. ${ }^{14}$ As the pseudostratified nasal epithelium is ciliated and has mucus-secreting goblet cells, it is less vulnerable to blistering compared to the stratified squamous keratinised and non-keratinised epithelium of the oral mucosa; as such, nasal intubation is preferable. ${ }^{13-15}$ Moreover, in comparison to an oral tube, a nasal tube is more easily secured without tape and provides a more spacious surgical field for dental treatment. ${ }^{13-15}$ Oesophageal strictures and dysphagia are common in patients with DEB and tend to increase the risk of gastro-oesophageal reflux, potentially affecting GA administration; ${ }^{18}$ however, this problem was not encountered in the current case.

\section{Conclusion}

DEB is a rare genetic disorder characterised by blistering and increased fragility of the skin and mucosal membranes following minor trauma. This report details the successful full dental clearance under GA of an adult with DEB which resulted in satisfactory healing and minimal scarring. The dental and anaesthetic care of DEB-affected individuals presents a challenge as they require special considerations and management to prevent complications.

\section{References}

1. Krämer SM. Oral care and dental management for patients with epidermolysis bullosa. Dermatol Clin 2010; 28:303-9. doi: 10.1016/j.det.2010.02.021.

2. Fine JD, Eady RA, Bauer EA, Bauer JW, Bruckner-Tuderman L, Heagerty A, et al. The classification of inherited epidermolysis bullosa (EB): Report of the Third International Consensus Meeting on Diagnosis and Classification of EB. J Am Acad Dermatol 2008; 58:931-50. doi: 10.1016/j.jaad.2008.02.004.

3. Fortuna G, Lozada-Nur F, Pollio A, Aria M, Cepeda-Valdes R, Marinkovich MP, et al. Patterns of oral mucosa lesions in patients with epidermolysis bullosa: Comparison and agreement between oral medicine and dermatology. J Oral Pathol Med 2013; 42:733-40. doi: 10.1111/jop.12094.

4. Wright JT, Fine JD, Johnson LB. Oral soft tissues in hereditary epidermolysis bullosa. Oral Surg Oral Med Oral Pathol 1991; 71:440-6. doi: 10.1016/0030-4220(91)90426-D.

5. Horn HM, Tidman MJ. The clinical spectrum of dystrophic epidermolysis bullosa. Br J Dermatol 2002; 146:267-74. doi: 10.1046/j.1365-2133.2002.04607.x

6. Uitto J, McGrath JA, Rodeck U, Bruckner-Tuderman L, Robinson EC. Progress in epidermolysis bullosa research: Toward treatment and cure. J Invest Dermatol 2010; 130:1778-84. doi: 10.1038/jid.2010.90.

7. Pillay E. Epidermolysis bullosa. Part 1: causes, presentation and complications. Br J Nurs 2008; 17:292-6. doi: 10.12968/ bjon.2008.17.5.28824

8. Abahussein AA, al-Zayir A, Mostafa W, Okoro AN Epidermolysis bullosa in the eastern province of Saudi Arabia. Int J Dermatol 1993; 32:579-81. doi: 10.1111/j.1365-4362.1993. tb05029.x.

9. Bruckner-Tuderman L. Dystrophic epidermolysis bullosa: Pathogenesis and clinical features. Dermatol Clin 2010; 28:107-14. doi: 10.1016/j.det.2009.10.020.

10. Intong LR, Murrell DF. Inherited epidermolysis bullosa: New diagnostic criteria and classification. Clin Dermatol 2012; 30:70-7. doi: 10.1016/j.clindermatol.2011.03.012.

11. Fortuna G, Aria M, Cepeda-Valdes R, Pollio A, MorenoTrevino MG, Salas-Alanís JC. Clinical features of gingival lesions in patients with dystrophic epidermolysis bullosa: A cross-sectional study. Aust Dent J 2015; 60:18-23. doi: 10.1111/ adj.12264.

12. Wright JT, Johnson LB, Fine JD. Development defects of enamel in humans with hereditary epidermolysis bullosa. Arch Oral Biol 1993; 38:945-55. doi: 10.1016/0003-9969(93)90107-W.

13. Lindemeyer R, Wadenya R, Maxwell L. Dental and anaesthetic management of children with dystrophic epidermolysis bullosa. Int J Paediatr Dent 2009; 19:127-34. doi: 10.1111/j.1365263X.2008.00940.x 
14. Esfahanizade K, Mahdavi AR, Ansari G, Fallahinejad Ghajari M, Esfahanizadeh A. Epidermolysis bullosa, dental and anesthetic management: A case report. J Dent (Shiraz) 2014; 15:147-52.

15. Wright JT. Comprehensive dental care and general anesthetic management of hereditary epidermolysis bullosa: A review of fourteen cases. Oral Surg Oral Med Oral Pathol 1990; 70:573-8. doi: 10.1016/0030-4220(90)90401-D.

16. Toda Y, Yokoyama M, Morimatsu H, Nakatsuka H, Takeuchi M, Katayama H, et al. General anesthesia in a patient with dystrophic epidermolysis bullosa. J Anesth 2006; 20:138-40. doi: 10.1007/s00540-006-0388-7.
17. Azrak B, Kaevel K, Hofmann L, Gleissner C, Willershausen B. Dystrophic epidermolysis bullosa: Oral findings and problems. Spec Care Dentist 2006; 26:111-15.

18. Fine JD, Mellerio JE. Extracutaneous manifestations and complications of inherited epidermolysis bullosa: Part I Epithelial associated tissues. J Am Acad Dermatol 2009; 61:367-84. doi: 10.1016/j.jaad.2009.03.052. 